\title{
An office chair to influence the sitting behavior of office workers
}

\author{
R.H.M. Goossens ${ }^{\mathrm{a}, \mathrm{b}^{*}}$, Netten, M.P. ${ }^{\mathrm{a}}$ and Van der Doelen, B. ${ }^{\mathrm{a}}$ \\ ${ }^{a}$ Department of Applied Ergonomics and Design, Delft University of Technology, Faculty of Industrial Design, \\ Landbergstraat 15, 2628 CE Delft, The Netherlands \\ ${ }^{\mathrm{b}}$ Department of Neuroscience, Erasmus MC, Dr. Molewaterplein 50, NL-3015 GE Rotterdam, The Netherlands
}

\begin{abstract}
Since the introduction of ergonomic guidelines in the design of office chairs, a lot of effort has been put in designing these office chairs accordingly. Because these features all have to be adjusted in different ways (mostly a knob underneath the seat surface), and because every office chair offers different solutions, often users do not use all of the adjustments, and thus do not use the office chair an the optimal ergonomic way. The aim of this paper is to study the influence of feedback on sitting habits of office workers in a field test during 4 weeks. 40 office workers were selected for this test (13 male, 27 female). They were divided in three groups. A control group, a group that received a sitting instruction and a group that received sitting instruction and feedback on their posture every hour that they sit. The results show that there is an effect in average increase in basic posture on both the group that received instruction and the group that received feedback. This effect decreases over time. There was no effect in the control group.
\end{abstract}

Keywords: sitting instruction, field study, smart technology

\section{Introduction}

Since the introduction of ergonomic guidelines in the design of office chairs, a lot of effort has been put in designing these office chairs accordingly [1]. This resulted in many different features in the office chairs, of which adjustment of seat height is the most well known. Because these features all have to be adjusted in different ways (mostly a knob underneath the seat surface), and because every office chair offers different solutions, often users do not use all of the adjustments, and thus do not use the office chair an the optimal ergonomic way [2].

This effect, the non-optimal use of the office chair, is reinforced by the actual use of the chair during office hours. Often the user has no clue about the postures that he or she adopted.

In order to influence the behavior of users, a Smart Chair was designed. With this chair, postures are calculated by means of 4 pressure sensors in the seat and 2 in the backrest. The chair detects every second the presence on the seat and calculates 7 different postures that can be adopted. Feedback about the posture that was adopted during one hour is presented by means of a label on the seat. Users will get a tactile stimulus to ask attention to their sitting postures.

The aim of this paper is to study the influence of the smart chair on sitting behavior of office workers in a field test during 4 weeks.

\section{Materials and methods}

40 office workers were selected for this test $(13$ male, 27 female, length 1.77 s.d. $0.17 \mathrm{~m}$, weight 74.7 s.d.2.1 kg). Inclusion criteria was that they had no history of back pain during the last 6 months and that they were working for at least 3 days a week during 4 consecutive weeks. During 4 weeks the sitting behavior of all of the groups is measured with technology in the Smart Chair. All participants started with a Control Week to measure regular sitting behavior. Office workers simply were given this new chair without any extra instructions. All users were aware

\footnotetext{
${ }^{*}$ Corresponding author: Tel: +31 15 2787179. Fax: +31 15 2787179. E-mail: r.h.m.goossens@tudelft.nl
} 
that the chair measures their sitting behavior. After this week they are divided in 3 groups that will re- ceive different interventions. All groups will continue this condition for another three weeks:

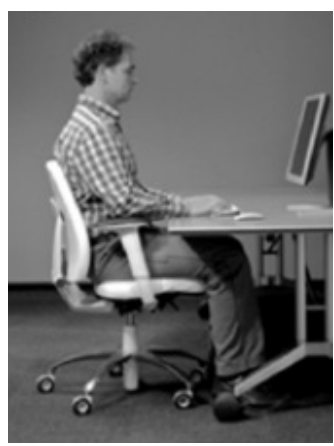

Fig. 1 The percentage of time that was spent in the basic posture can be measured by means of the chair. Feedback about the posture that was adopted during one hour is presented by means of a label on the seat.

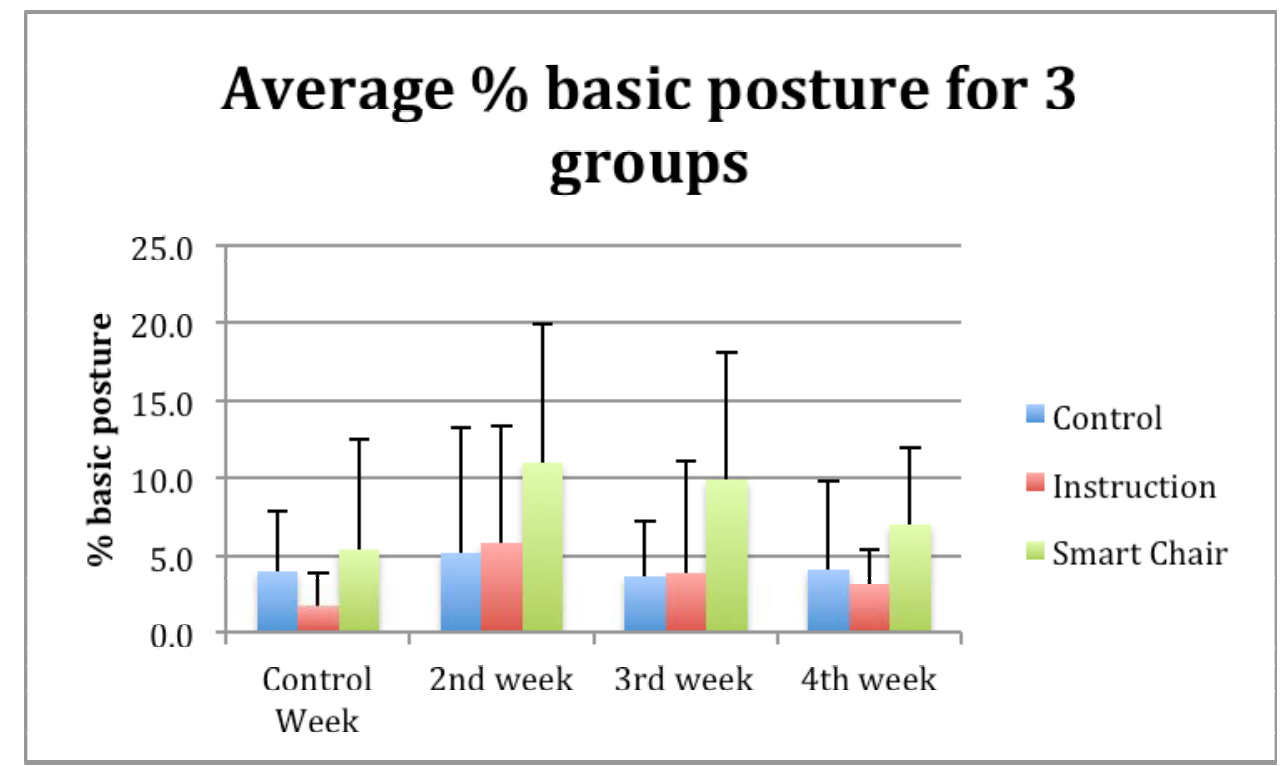

Fig. 2 The average $\%$ of time that the basic posture was adopted during a week for all the subjects in a group. As can be seen, the standard deviation is rather large, because of the different sitting habits of each subject. It can also be seen that there is an increase of the average percentage of basic posture after the control week for as well the group that received an instruction and the group that received feedback of their sitting habits by means of a label on the smart chair. In week 2 the average effect is largest for the smart chair and seems to reduce in the weeks after that.

- Group A (Smart Chair) $(\mathrm{n}=15)$ : receives an instruction on the optimal use of the chair in the beginning of the 2 nd week and receives feedback on their posture every hour that they sit from week 2 to 4

- Group B (Instruction) $(\mathrm{n}=14)$ : receives an instruction on the optimal use of the chair in the 2 nd week, but does not get feedback;

- Group C (Control) $(\mathrm{n}=11)$ : is a control group, they get no feedback and no instruction.
The percentage of time that was spent in the basic posture $\mathrm{P}_{\mathrm{B}}$ (Figure 1) during a week, per subject was used for statistics. A Wilcoxon Signed Ranks Test was used the test on a significant difference between the control week (1st week) and the other weeks.

$$
\begin{aligned}
& \text { H0: } P_{B, \text { Control week }}=P_{B, \text { week } n} \\
& \text { H1: } P_{B, \text { Control week }} \neq P_{B \text {,week } n}(n=2,3,4)
\end{aligned}
$$


A Kruskal-Wallis was used to see if there were significant differences between the groups during the control week.

The level of significance for both tests was 0.05 .

Table 2

Average percentage of time per group per week that the subjects were sitting in a basic posture

\begin{tabular}{lllllll} 
& \multicolumn{6}{c}{$\%$ time in basic posture (in brackets s.d.) } \\
\hline & Control & \multicolumn{2}{c}{ Instruction } & \multicolumn{2}{c}{ Smart Chair } \\
\hline Control Week & 4.0 & $(3.9)$ & 1.8 & $(2.1)$ & 5.4 & $(7.1)$ \\
2nd week & 5.2 & $(8.1)$ & 5.8 & $(7.6)$ & $11.0 \quad(8.9)$ \\
3rd week & 3.7 & $(3.5)$ & 3.9 & $(7.2)$ & 9.9 & $(8.2)$ \\
4th week & 4.1 & $(5.7)$ & 3.1 & $(2.3)$ & 7.0 & $(5.0)$
\end{tabular}

\section{Results}

Because of difficulties with the collection of data 2 subjects in the Instruction Group were excluded out of the tests. Figure 2 and table 2 -shows the final results. As can be seen, the standard deviation is rather large, because of the different sitting habits of each subject. An effect can be seen between the three different groups. The control group does not change its sitting behavior in the $2 \mathrm{nd}$, 3rd and 4th week com-

\section{Discussion}

The results of this research on the influence of feedback on the behavior of office workers seem promising. As was expected, it was shown that an instruction on how to use the office chair has an effect on the behavior. This study shows that users can change their behavior on the basis of feedback too. During the first week after the feedback the percentage that the office workers of the Smart Chair group spend in a basic posture doubled, on average, from $5.4 \%$ to $11 \%$.

pared to the control week, whereas the other two groups do show an effect.

There was no difference between the groups in the control week (Kruskal-Wallis test $\mathrm{P}=0.257$ ).

The effect is significant in the 2nd week for the Smart Chair group $(p=0.011)$ and the Instruction group $(p=0.010)$. In the $3 \mathrm{rd}$ week this effect is not significant for the Smart Chair group $(\mathrm{p}=0.088)$ nor for the Instruction group ( $\mathrm{p}=0.594)$. In the 4 th week the effect is not significant for the Smart Chair group. $(p=0.21)$ but it is for the Instruction group $(p=0.021)$.

As expected too, the data also confirmed that there is a large difference between office workers in their sitting behavior. The current study with the Smart Chair can be seen as a first step to influence this behavior, and will be complemented with a longer follow up study in the future.

\section{References}

[1] Grieco, A., 'Sitting posture: an old problem and a new one', Ergonomics, 29 (1986), 345-62.

[2] Groenesteijn, L., et al., 'Effects of differences in office chair controls, seat and backrest angle design in relation to tasks', Applied ergonomics, 40 (2009), 362-70. 